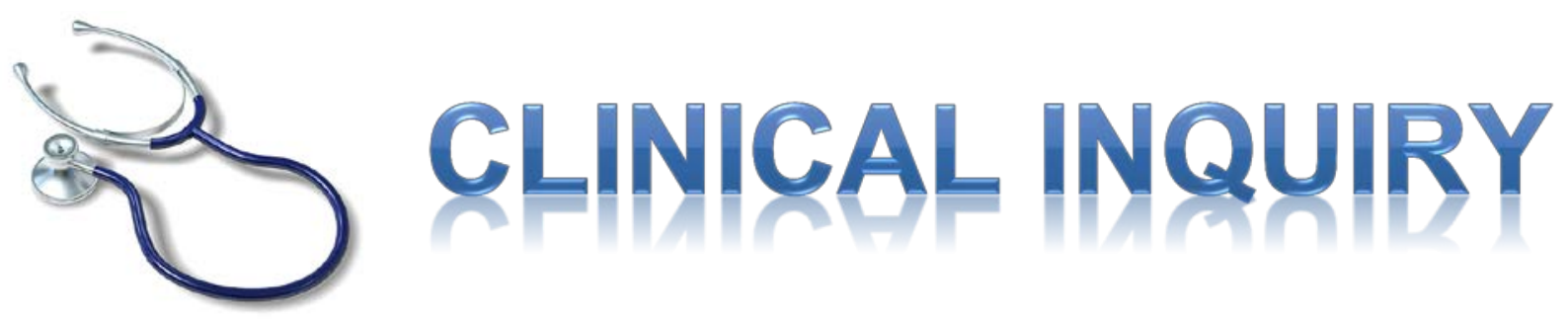

\title{
Efficacy of Local Corticosteroid Injection for Carpal Tunnel Syndrome
}

\author{
Brett Hoffecker, M.D., Aaron Hightower, M.D., Jessica Jarvis, M.D., Douglas Lewis, M.D., \\ Jennifer Wipperman, M.D., MPH \\ University of Kansas School of Medicine-Wichita \\ Department of Family and Community Medicine \\ Family Medicine Residency Program at Via Christi
}

\section{Clinical Question}

In patients with carpal tunnel syndrome (CTS), do local corticosteroid injections provide symptomatic relief beyond one month?

\section{Evidence-Based Answer}

Local steroid injections provide symptomatic relief for greater than one month in patients with CTS (Strength of Recommendation (SOR) A) and up to 12 months (SOR B). Local steroid injection also decreases the need for surgery at one year for patients with non-severe CTS (SOR B).

\section{Methodology}

A search was conducted on relevant studies published since a 2007 Cochrane review ${ }^{1}$ of local steroid injection for CTS. PubMed and the Cochrane Database were searched from January 2007 to November 2014. Search terms included steroid injection, corticosteroid injection, injection, carpal tunnel, and carpal tunnel syndrome. Thirty-eight relevant studies were found, and of these, eight relevant studies have been published since the Cochrane review and were included in this review. Participants included adults ages 18 and older diagnosed with CTS. CTS may have been defined using clinical or electrodiagnostic criteria. Patients with all severities of CTS were included.

\section{Evidence Summary}

Carpal tunnel syndrome is a condition frequently managed by primary care providers, and is the most common compressive neuropathy of the upper extremity. ${ }^{2}$ Non-surgical management is the initial treatment strategy for most patients with mild to moderate CTS. Conservative treatment also may be used as a bridge for patients who wish to avoid or delay surgery. Multiple non-surgical options exist, including splinting, oral corticosteroids, and physical therapy. Conservative treatment options generally provide relief from two to 12 weeks. ${ }^{3}$ Local corticosteroid injection is a simple, quick office procedure that may provide longer benefit. While a 2007 Cochrane review of local corticosteroid injection for CTS only found evidence for 
symptomatic benefit up to one month after injection, ${ }^{1}$ more recent evidence suggests benefit lasting from three months to one year. In addition, corticosteroid injection may delay the need for surgery.

Corticosteroid injection leads to symptomatic improvement in CTS for greater than one month. In a double-blinded, randomized trial, adults with carpal tunnel syndrome of moderate severity were treated with a single injection of either $80 \mathrm{mg}$ of methylprednisolone, $40 \mathrm{mg}$ methylprednisolone, or placebo. ${ }^{1}$ Primary end points included change in CTS symptom score (range 1 through 5, 5 being most severe) at ten weeks and one year, and rate of surgery at one year. Patients who received $80 \mathrm{mg}$ or $40 \mathrm{mg}$ of methylprednisolone noted greater improvement in symptoms compared to those who received placebo (difference in change from baseline, -0.64 [95\% CI, -1.06 to $-0.21 ; p=0.003$ ] and -0.88 [95\% CI, -1.30 to -0.46 ; $p<0.001$ ], respectively). However, there was no difference at one year in symptom score.

In a single-blinded, randomized trial, 46 adults with CTS of moderate severity were treated with either a single ultrasound-guided or palpation-guided injection of $40 \mathrm{mg}$ methylprednisolone. ${ }^{2}$ Primary end points included change in CTS symptom and function scores (range 1 through 5, 5 being most severe) at 6 and 12 weeks. Patients experienced relief of symptoms and return of function at six weeks of follow up, with no difference between groups. At 12 weeks, the ultrasound-guided group had a greater reduction in symptoms, but there was no difference in function compared to palpation-guided group (mean symptom score $1.30+/-0.45$ vs $1.67+/-0.73, \mathrm{p}<0.001$; mean function score $1.36+/-0.49$ vs $1.86+/-1.09, \mathrm{p}=0.298$ ). It is theorized that an ultrasound-guided approach leads to more accurate placement of injection, and therefore possibly greater efficacy.

A randomized, placebo-controlled trial of 57 patients with CTS randomized 90 wrists to triamcinolone acetonide injection (40 mg), 1\% procaine $\mathrm{HCl}$ injection, or normal saline injection. ${ }^{3}$ A previous study found that local anesthetic injection alone can lead to improvement in carpal tunnel syndrome, leading the investigators to compare local anesthetic, local steroid, and saline injections. ${ }^{4}$ Outcomes included both symptomatic scores and electrophysiologic measurements. At two and six months of follow-up after a single injection, treatment with triamcinolone injection showed significant improvement in electrophysiologic and symptomatic scores compared to placebo ( $\mathrm{p}<0.05$ ). While no difference was found between the triamcinolone and procaine groups, there was a trend toward greater improvement from baseline in symptomatic and electrophysiologic scores for the steroid group versus procaine group. Lack of significance could be due to small sample size.

Corticosteroid injection leads to symptomatic improvement in CTS for up to 12 months. In a trial of 163 wrists with CTS, wrists were randomized to either surgical decompression or paramethasone acetonide $20 \mathrm{mg}$ injection and followed for 24 months. ${ }^{5}$ In the steroid group, an additional injection was allowed if symptoms had not resolved completely, and $84 \%$ of participants in the injection group received a second injection. At three months follow-up, symptoms were improved significantly in the injection group compared to the surgery group. At 6, 12 and 24 months, there was no difference in percentage of patients achieving at least a 50\% improvement in symptom and functional scores. However, at 24 months, surgery was more effective than injection in those achieving $70 \%$ improvement in functional scores $(60.0 \%$ vs $44.6 \%$ respectively; $\mathrm{p}=0.049$ ).

A trial of 69 patients with CTS in a general practice setting randomized participants to either triamcinolone acetonide $10 \mathrm{mg}$ or normal saline injection and observed symptomatic and functional scores for 12 months. ${ }^{6}$ Up to two steroid injections were allowed. Steroid injection 
was significantly more effective than placebo for improvement of symptoms and function at 3, 6 , and 12 months, with greater than 30\% of patients reporting symptomatic improvement at 12 months.

In a prospective cohort study, 120 patients with CTS underwent up to three injections with 40 mg methylprednisolone and were followed for one year. ${ }^{7}$ Forty-six patients (38\%, 95\% CI 3047\%) had significant improvement at one year.

In a retrospective cohort study by Visser et al. ${ }^{8}, 273$ with patients with CTS were treated with up to two local injections of $40 \mathrm{mg}$ methylprednisolone. Symptomatic improvement was seen in $63 \%$ of patients at 6 months, $48 \%$ at 12 months, and 34\% for more than 18 months.

Patients with moderate CTS who have a corticosteroid injection are less likely to undergo surgical decompression at one year. In the trial by Atroshi et al. ${ }^{4}$ noted above, patients injected with $80 \mathrm{mg}$ of methylprednisolone were less likely to have surgery at one year compared to placebo (odds ratio, 0.24 [CI, 0.06 to 0.95]; $\mathrm{p}=0.042$ ), and when time to surgery was analyzed, both the $80 \mathrm{mg}$ and $40 \mathrm{mg}$ methylprednisolone groups had a lower likelihood of surgery (hazard ratio, 0.46 [CI, 0.27 to $0.77 ; p=0.003$ ] and 0.57 [CI, 0.35 to $0.94 ; p=0.026$ ], respectively). In the retrospective cohort study noted above by Visser et al. ${ }^{10}$, the median time until surgery was 15 months for mild, 5 months for moderate and 4.5 months for severe CTS $(p=0.02)$. Finally, a survivorship analysis of 824 patients receiving a single injection of $20 \mathrm{mg}$ methylprednisolone for CTS found that the rate of surgery was $14.5 \%$ after one year and $33.2 \%$ after five years. ${ }^{9}$

\section{Recommendations from Others}

The American Academy of Orthopaedic Surgeons (AAOS) recommends conservative treatment for patients with mild to moderate carpal tunnel syndrome before considering surgery, and considers local steroid injection to be effective treatment. ${ }^{10}$ The American College of Occupational and Environmental Medicine gave a level A recommendation for carpal tunnel injections for subacute or chronic CTS. ${ }^{11}$ Specific recommendations concerning length of treatment and number of injections are lacking, however in general, most wait 12 weeks before repeating intra-articular steroid injections.

\section{Conclusion}

Current evidence shows benefit from local steroid injection for greater than one month in patients with CTS. Benefit can last beyond 12 months and limit the need for surgery. Interestingly, studies that allowed for repeat injection tended to show greater efficacy in the long-term. Time to second injection in the above studies ranged from 1 week to 6 months, with most patients requiring injection before 6 months $^{7}$. Further study is needed to compare frequency and timing of steroid injections for carpal tunnel syndrome.

\section{References}

1 Atroshi I, Flondell M, Hofer M, Ranstam J. Methylprednisolone injections for the carpal tunnel syndrome: A randomized, placebo-controlled trial. Ann Intern Med 2013; 159(5):309-317. PMID: 24026316.

${ }^{2}$ Ustun N, Tok F, Yagz AE, et al. Ultrasound-guided vs. blind steroid injections in carpal tunnel syndrome: A single-blind randomized prospective study. Am J Phys Med Rehabil 2013; 92(11):999-1004. PMID: 23811617. 
${ }^{3}$ Karadas O, Tok F, Akarsu S, Tekin L, Balaban B. Triamcinolone acetonide vs procaine hydrochloride injection in the management of carpal tunnel syndrome: Randomized placebocontrolled study. J Rehabil Med 2012; 44(7):601-604. PMID: 22674243.

${ }^{4}$ Karadas O, Tok F, Ulas UH, Odabaşi Z. The effectiveness of triamcinolone acetonide vs. procaine hydrochloride injection in the management of carpal tunnel syndrome: aA double-blind randomized clinical trial. Am J Phys Med Rehabil 2011; 90(4):287-292. PMID: 21273900.

5 Ly-Pen D, Andreu JL, Millan I, de Blas G, Sanchez-Olaso A. Comparison of surgical decompression and local steroid injection in the treatment of carpal tunnel syndrome: 2-year clinical results from a randomized trial. Rheumatology 2012; 51(8):1447-1454. PMID: 22467087.

6 Peters-Veluthamaningal C, Winters JC, Groenier KH, Meyboom-de Jong B. Randomised controlled trial of local corticosteroid injections for carpal tunnel syndrome in general practice. BMC Fam Practice 2010; 11:54. PMID: 20670438.

7 Berger M, Vermeulen M, Koelman JH, van Schaik IN, Roos YB. The long-term follow-up of treatment with corticosteroid injections in patients with carpal tunnel syndrome. When are multiple injections indicated? J Hand Surg Eur 2013; 38(6):634-639. PMID: 23221180.

${ }^{8}$ Visser LH, Ngo Q, Groeneweg SJ, Brekelmans G. Long term effect of local corticosteroid injection for carpal tunnel syndrome: A relation with electrodiagnostic severity. Clin Neurophysiol 2012; 123(4):838-841. PMID: 21962473.

9 Jenkins PJ, Duckworth AD, Watts AC, McEachan JE. Corticosteroid injection for carpal tunnel syndrome: A 5-year survivorship analysis. Hand 2012; 7(2):151-156. PMID: 23730233.

${ }^{10}$ Keith MW, Masear V, Chung KC, et al. American Academy of Orthopaedic Surgeons clinical practice guideline on the treatment of carpal tunnel syndrome. J Bone Joint Surg Am 2010; 92(1):218-219. PMID: 20048116.

${ }^{11}$ Carpal Tunnel Syndrome. In: Hegmann KT. (Ed.) Occupational medicine practice guidelines. Evaluation and management of common health problems and functional recovery in workers. Third edition. Elk Grove Village, IL: American College of Occupational and Environmental Medicine, 2011. ISBN 1-883595-42-8.

Keywords: carpal tunnel syndrome, glucocorticoids 


\section{Appendix}

(Adapted from American Family Physician*)

\section{Strength of recommendation}

A

B

C

*http://www.aafp.org/dam/AAFP/documents/journals/afp/sortdef07.pdf

** Patient-oriented evidence measures outcomes that matter to patients: morbidity, mortality, symptom improvement, cost reduction, and quality of life.

Disease-oriented evidence measures intermediate, physiologic, or surrogate end points that may or may not reflect improvements in patient outcomes (e.g., blood pressure, blood chemistry, physiologic function, pathologic findings). 